\title{
Relationship with Lung Carcinoma in the Patients with Anthracosis by Detection of Expression of P16 and MUC1 Genes
}

\author{
Mona Ghashghaee ${ }^{1}$, Naghmeh Bahrami ${ }^{2}$, Tahereh Naji ${ }^{3}$, Abdolreza Mohamadnia ${ }^{4,5}$
}

${ }^{1}$ Department of Molecular and Cellular Sciences, Faculty of Advanced Sciences and Technology, Pharmaceutical Sciences Branch, Islamic Azad University, Tehran, Iran. ${ }^{2}$ Craniomaxillofacial Research Center, Tehran University of Medical Sciences, Tehran, Iran; Department of Oral and Maxillofacial Surgery, School of Dentistry, Tehran University of Medical Sciences, Tehran, Iran. ${ }^{3}$ Department of Basic Sciences, Faculty of Pharmacy, Pharmaceutical Sciences Branch, Islamic Azad University, Tehran, Iran. ${ }^{4}$ Virology Research Center, National Research Institute of Tuberculosis and Lung Diseases (NRITLD), Shahid Beheshti University of Medical Sciences, Tehran, Iran. ${ }^{5}$ Department of Biotechnology, School of Advanced Technologies in Medicine, Shahid Beheshti University of Medical Sciences, Tehran, Iran.

\begin{abstract}
Introduction and objective: Anthracosis is a pneumoconiosis caused by coal dust. The term "pneumoconiosis" refers to an occupational lung disease caused by long-term inhalation of dust. To achieve more efficient diagnosis and treatment methods, changes in the expression of MUC1 and P16 genes in the patients with anthracosis and their relationship with none-small cells lung carcinoma were examined. Methodology: Thirty anthracosis samples (center of the lesion) and thirty healthy samples (edges of the lesion) were collected from anthracosis patients diagnosed by a specialist. The patients signed a written letter of consent beforehand. The expression of MUC1 and P19 biomarkers was measured through Real-Time PCR. The relationship of the expression of biomarkers with tumor staging and lung carcinoma stage was examined. Results: The two groups in the study were compared using t-test. P16 marker was positive at the center and edges of lesions in seven (out of 30) and 18 (out of 30) cases respectively. There was a significant difference between positive P16 markers at the center and edges of the lesions ( $\mathrm{p}$-value $<0.001$ ). MUC1 marker was positive at the center of lesions in 21 cases out of 30 (sensitivity $=70 \%$ ) and it was positive at the edges of lesions in seven cases out of 30 . In terms lesions ( $\mathrm{p}$-value $<0.001$ ). Conclusion: The results indicated a relationship between anthracosis and lung carcinoma. Clearly, the positive cases of P16 genes at the edges of lesions were higher than that at the center; while the positive cases of MCU1 gene at the center of lesions were higher than that at the edges. Further studies with larger sample groups are needed to provide more evidence.
\end{abstract}

Keywords: Anthracosis disease- lung carcinoma- MUI1 Biomarker- P16 Biomarker

Asian Pac J Cancer Care, 2 (4), 59-63

\section{Introduction}

The term "anthracosis" is comprised of "antrac" for coal or carbon and "osis" for a mode or a condition. Technically, anthracosis is featured with black plaques on bronchus mucus, pulmonary parenchyma, respiratory bronchial, which can be diagnosed through bronchoscopy [1]. The disease is called bronchial anthracofibrosis (BAF) when it causes deformity and lumen bronchus stenosis [2].In addition, damage to bronchus mucus disrupts
Submission Date: 05/15/2017Ａcceptance Date: 08/11/2017

cilia sweeping function that leads to other infections like pulmonary tuberculosis [3].

Some of the pathophysiology authors have mentioned bronchial stenosis, large lymph nodes with or without calcification and high risk of malignant tuberculosis as the results of inhalation of biomass smock [4]. There is no record of anthracosis plaques, bronchial stenosis, and deformation in the individual with COPD (with smoking history); thereby, anthracosis cases diagnosed through bronchoscopy are not considered

Corresponding Author:

Dr. Abdolreza Mohamadnia

Virology Research Center, National Research Institute of Tuberculosis and Lung Diseases (NRITLD), Shahid Beheshti University of Medical Sciences, Tehran, Iran.

Department of Biotechnology, School of Advanced Technologies in Medicine, Shahid Beheshti University of Medical Sciences, Tehran, Iran.

Email: mohamadnia.ar@gmail.com 
as a COPD case [5].

In general, anthracosis is mostly initiated from respiratory bronchiole, which is a part of respiratory zone[6]. Coughing and dyspnea are of the most common symptoms of BAF and anthracosis (Table1) along with sibilant rhonchi heard through physical examination [7]. In some cases, anthracosis is misdiagnosed as tuberculosis, lung cancer, or pneumonia [8].

Cytology studies on the lung tissue have shown that inter-bronchial anthracosis and lymphadenopathy meditation are coincident with the early primitive adenocarcinoma. Thereby, one may conclude that there is a significant relationship between anthracosis and lung carcinoma [9].

Lung carcinoma is the most common type of cancer in men all around the world [10-14]. It includes small cell lung carcinoma (SCLC) and non-small cell lung carcinoma (NSCLC) [10]. The former is highly lethal and there is a high mortality rate among SCLC patients [15]. Today, many researchers work on finding new noninvasive biomarkers to assess, screen, categorize, and survey cancers [16].

P16 is a tumor suppressor protein with proved anti-tumor properties [17].There are evidence of the effectiveness of expression of P16 protein in uncontrolled cellular proliferation and tumors growth. In addition, deactivation of P16 gene has been observed in $25-70 \%$ of lung carcinoma cases. Thereby, there is a relationship between changes in P16 gene activation and tumor occurrence [18].

Mucin 1 (MUC1) is a membrane glycoprotein comprised of two subunits of MUC1-N (extracellular mucin subunit) and MUC1-C (transmembrane mucin subunit) that functions as an oncoprotein [19]. The expression of MUC1 differs in normal and cancerous conditions. Over-expression of MUC1 has been reported in several types of cancers like lung, pancreas, colorectal, cervical, and prostate cancers [20-22].

\section{Materials and Methods}

Real-time (RT) PCR technique was used to survey the expression of $\mathrm{P} 16$ and MUC1 genes in 30 patients in Masih Daneshvari Hospital-Tehran. Anthracosis cases were confirmed by a specialist. Tissue samples were collected from the center and edges of black lesions of anthracosis. RNA of the sample was extracted and then transformed into CNDA on which RT PCR technique was implemented. It is notable that this process was carried out as a trial check.

\section{RNA extraction}

The extraction process was carried out using Cinna pure RNA (cat.No.:PR891620-S). So that, one 20-25mg piece of the sample was crushed at first and then $400 \mu 1$ lysing solution was added to the kit, mixed, and homogenized.

Afterward, Precipitation solution was added to the homogenized mixture and the obtained solution was centrifuged on an extraction column.

Wash buffers I \& II were used in the column and centrifuge respectively. Afterward, $50 \mu 1 \mathrm{RNase}$ free water was added to the column and pure RNA was extracted. Quality of RNA was examined using a nano-drop device.

\section{cDNA development}

cDNA was developed based on the extracted mRNA using Viva 2-steps RT-PCR Kit (Cat No.RTPL12). The kit is featured with high sensitivity and capability to proliferate any DNA out of pattern RNA.

\section{Specific primers design}

Specific primers were designed for each biomarkers using AllelelID6 and then ordered to the producer. All primers had proper $\mathrm{Tm}$ and $\mathrm{C}+\mathrm{G}$ rates and were free of internal loop and hairpin series. In addition, the primers were examined in terms of dimerization.

\section{$R T-P R C$ reaction}

The reaction was implemented using GinnaGreen qPCR Mix.2X (Cina Colon Co- Cat No. MM2041). The kit uses a fluorescence emitter known as EvaGreen, which illuminates florescence light after being attached to two-strand DNA and then the light is measured by PCR.

The elements needed for the reaction were prepared in a master-mix kit. The elements of RT-PCR reaction included:

a. Sequence of the pattern $(2 \mu \mathrm{l})$;

b. Master-mix $(4 \mu 1)$;

c. Primer (optimum concentration was determined

in the primary set up tests); and

d. Deionized distilled water to increase the volume of reaction to $20 \mu 1$.

It is notable that gene reference 18 srRNA was used for internal control and the results of each reaction were interpreted based on the amplification and melt curves.

\section{Data analyses}

The findings were analyzed in SPSS V20 using mean, standard deviation, and t-test.

\section{Results}

The study was carried out using 30 tissue samples collected from six women and 24 men. The average age of the patients was $48 \pm 10.5$. 18sRNA gene was used as the reference gene given that the comparison of mean "ct" values at the center and edges of lesions indicated no significant difference ( $\mathrm{p}$-value $=0.263$ ).

P16 gene was positive at the center of lesions (affected) in 7 cases out of 30 . Value of the biomarker at edges of the lesion (healthy) was positive in 18 cases out of 30 . Comparison of positive cases of P16 gene at the center and edges of lesions showed no significant difference. Positive cases of the biomarker in the affected and healthy groups were compared using two-sample binomial, which indicated no significant difference between the two groups (p-value $<0.001$ ). 
Table 1. The Primers Used in RT-PCR

\begin{tabular}{lccc}
\hline Parameters & P16 & MUC1 & 18s rRNA \\
\hline F primer & ACCCTGGATGTCCTCTATGG & GTGCCCCCTAGCAGTACCG & GTAACCCGTTGAACCCCATT \\
Primer length & 20 & 19 & 20 \\
R primer & CAGGCATAGGTCCCGTTATTA & GACGTGCCCCTACAAGTTGG & CCATCCAATCGGTAGTAGCG \\
Primer length & 21 & 20 & 20 \\
\hline
\end{tabular}

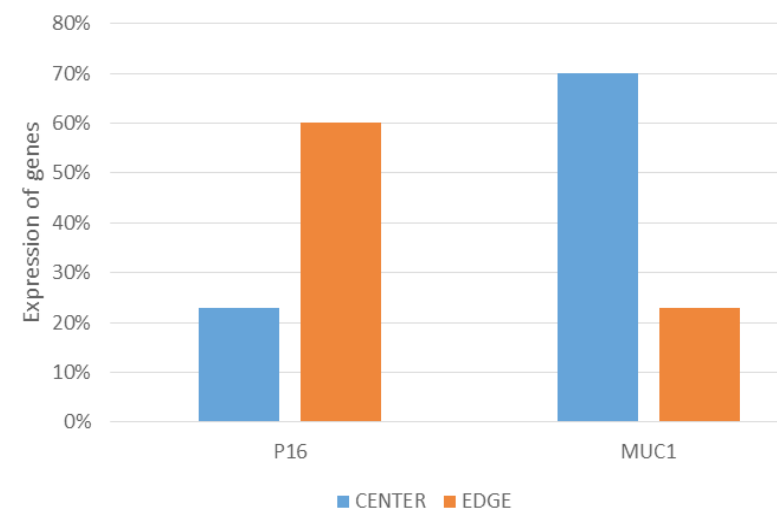

Figure 1. Evaluation of MUC1 and P16 mRNA Expression in the Center and Edges of the Lesions by RT PCR.

With regard to MUC1 gene, there were 21 positive cases at the center of the lesion (sensitivity $=70 \%$ ) and 7positive cases at the edges of the lesion (healthy). Comparison of positive MUC1 genes at the center and edges of lesions showed a significant difference. Positive biomarkers in the affected and healthy samples were compared using Two-sample binomial test and the result confirmed a significant difference between the two groups (p-value $<0.001$ ) ( Figure 1).

Clearly, positive cases of P16 gene at the edges of the lesion was higher than the center, however, in the case of MUC1 gene, positive cases at the center of lesions was higher than the edges. In general, the risk of NSCLC in anthracosis patients is high; a fact to be taken into account in treatment and follow up programs. To obtain more accurate results with higher sensitivity, the experiments should be conducted with three iterations.

Expression of P16 and MUC1 genes at the center and edges of lesions

"ct" value of each sample was determined at first and then the relative difference of expression of the biomarkers was measured and compared. To this end, the $\Delta \Delta \mathrm{Ct}$ method was used for MUC1 and P16 biomarkers and then the difference between the biomarkers in both groups was obtained by calculating 2 to the power $-\Delta \Delta \mathrm{Ct}$.

Table 2. Temperatures and Time of RT-PCR Reaction

\begin{tabular}{lccc}
\hline Cycle step & Temperature & Time & Cycles \\
\hline Initial denaturation & $95^{\circ} \mathrm{C}$ & $10 \mathrm{~min}$ & 1 \\
Denaturation & $95^{\circ} \mathrm{C}$ & $10 \mathrm{~s}$ & \\
Annealing & $65-60^{\circ} \mathrm{C}$ & $60 \mathrm{~s}$ & 45 \\
Elongation & $72^{\circ} \mathrm{C}$ & $20 \mathrm{~s}$ & \\
\hline
\end{tabular}



Figure 2. Difference in Expression of P16, MUC1Genes at the Center and Edges of Lesions

Value of $\Delta \Delta \mathrm{Ct}$ for $\mathrm{P} 16$ biomarker was -0.9 and 2 to the power of $-\Delta \Delta \mathrm{Ct}$ yielded the difference between the biomarkers. On average, the number of primary copies of the markers at the edges was 1.8times more than that at the center.

Value of $\Delta \Delta \mathrm{Ct}$ for MUC1 biomarker was -0.8 and 2 to the power of $-\Delta \Delta \mathrm{Ct}$ yielded the difference between the biomarkers. On average, the number of primary copies of the markers at the center was 2.8times more than that at the edges Figure 2.

\section{Discussion}

In general, anthracosis is characterized by black plaques on bronchial mucus, pulmonary parenchyma, and respiratory bronchial and it is diagnosed through bronchoscopy [23]. Although the disease is a simple indicator of air pollution and smoking habit, it is not easy to estimate the level of dust and black particles [24]. Exposure to air pollution in urban areas and direct occupational exposure to smock through inhalation are the risk factors of anthracosis [25].

Studies have confirmed the relationship between anthracosis and clinical specification of pulmonary adenocarcinoma [25]. Among different tumor markers, it appears that mRNA biomarkers are the best choice as small a volume of them can be spotted through RT PCR method - a method with high accuracy. In light of this, MUC1 and P16 biomarkers were under focus in this study.

The results of expression of $\mathrm{p} 16$ protein have been observed in uncontrolled cellular proliferation and tumor growth and deactivation of the P16 gene has been reported in $25-70 \%$ of pulmonary carcinoma cases. Therefore, there is a relationship between changes in the expression of $\mathrm{P} 16$ gene and the development of tumors [18]. In addition, 
over-expression of MUC1 has been reported in many cancers such as pulmonary, pancreas, colorectal, cervical, and prostate cancers [21].

Hongyan Wand et al. (2017) surveyed the relationship between expression P16 gene (tumor suppressor) and metastasis of lymphatic nodes. They also studied prognosis of ovarian cancer. Their results showed a significant decrease in the positive expression of P16 gene in cancerous tissue of ovarian in comparison with the unaffected tissue. In addition, they reported that the positive expression of p16 in cancerous ovarian tissue with metastasis of cancerous lymph decreased in comparison with the tissue without metastasis [26]. Consistently, our results showed a decrease in the expression of the gene at the center of lesions on the pulmonary tissue of anthracosis patients.

In another study in 2017, the expressions of P27 and P16 proteins in 83 cases of larynx lesions (32 larynx papilloma and 51 carcinoma cases) were compared. Immunohistochemistry reaction was used to diagnose P27 and P16 expression levels and the results indicated a decrease in the expression of P27 and P16 in cancerous cells comparing with papilloma. In addition, there was a cytoplasmic expression of p16 in cancerous cases and no expression in papilloma cases. The study showed that a change in the expression of P27 and p16 proteins might be a valuable indicator of larynx squamous cells carcinoma [27]. Consistently, our results showed a decrease in the expression of P16 in cancerous cases, which is due to tumor suppression nature of the biomarker so that its expression decreases in the most types of cancers.

In another paper published in Korean Journal of Pathology on MUC1 in 2011, the expressions of MUC1 and MUC4 genes in NSCLC patients were examined to determine the prognoses. The article reported a significant relationship between the expressions of MUC4 and MUC1 and that the high expression of the two genes has a significant relationship with adenocarcinoma [28]. The study was based on immunohistochemistry on 165 pathology cases, and consistent with the results of the present study, the oncogenic role of MUC1 and the increase of its expression in NSCLC cancer was highlighted. Still, the increase in the expression of this biomarker, in the present study, was observed in the tissue samples collected from the center of lesions.

In general, P16 is a regulator protein of cell cycle and its expression is attenuated in cancerous cells. Therefore, cells become cancerous when the expression of protein decreases. Given that the expression of MUC1 gene in NSCLC cancers increases, one may conclude that black lesions of anthracosis lead the cells toward the development of NSCLC.

To have more reliable results and evidences, future studies with larges sample sizes are needed. In addition, surveying other biomarkers of lung carcinoma can shed more light on the topic and improve the accuracy of cancer screening tests.

\section{References}

1. TOUHIDI M KM, Ataran D, et al Tuberculous bronchostenosis presenting as anthracofibrosis.(2002).

2. Han F, Yang T, Song L, Zhang Y, Li H, Guan W, et al. Clinical and pathological features and imaging manifestations of Bronchial anthracofibrosis: the findings in 15 patients. Chinese medical journal. 2013;126(14):2641-6.

3. Park JH, Lee S-H, Bae W-Y, Park DH, Cho HD, Park $\mathrm{S}-\mathrm{H}$, et al. Esophageal anthracosis with endobronchial tuberculosis: case report. Gastrointestinal endoscopy. 2006;64(6):1022-4.

4. Kim YJ, Jung CY, Shin HW, Lee BK. Biomass smoke-induced Bronchial anthracofibrosis: presenting features and clinical course. Respiratory medicine. 2009;103(5):757-65.

5. Heidarnazhad H. Anthracosis in Iran, unanswered questions. Archives of Iranian medicine. 2012;15(3):124.

6. Anuradha Murty D, Das D. Pulmonary tuberculosis with anthracosis: an unusual diagnosis by fine needle aspiration cytology. Acta cytologica. 1993;37(4):639-40.

7. Chung MP, Lee KS, Han J, Kim H, Rhee CH, Han YC, et al. Bronchial stenosis due to anthracofibrosis. Chest. 1998;113(2):344-50.

8. Hanahan D, Weinberg RA. The hallmarks of cancer. cell. 2000;100(1):57-70.

9. Ochs M, Nyengaard JR, Jung A, Knudsen L, Voigt M, Wahlers T, et al. The number of alveoli in the human lung. American journal of respiratory and critical care medicine. 2004;169(1):120-4.

10. Moore RG, McMeekin DS, Brown AK, DiSilvestro P, Miller $\mathrm{MC}$, Allard WJ, et al. A novel multiple marker bioassays utilizing HE4 and CA125 for the prediction of ovarian cancer in patients with a pelvic mass. Gynecologic oncology. 2009;112(1):40-6.

11. Karimi S, Mohamadnia A, Nadji SA, Yadegarazari R, Khosravi A, Bahrami N, et al. Expression of two basic mRNA biomarkers in the peripheral blood of patients with non-small cell lung cancer detected by real-time rt-PCR, individually and simultaneously. Iranian biomedical journal. 2015;19(1): 17 .

12. Karimi S BN, Sharifi K, Daustany M, Baghbani-Arani F, Kazempour $\mathrm{M}$, et al. Investigating gene expression level of MUC1 and CEA in pleural fluid of NSCLC lung cancer patients with the real-time RT-PCR method. MINERVA PNEUMOLOGICA. 2017; 56(1):18-24.

13. Ghadimi K, Bahrami N, Fathi M, Farzanegan B, Naji T, Emami M, et al. Diagnostic value of LunX mRNA and CEA mRNA expression in pleural fluid of patients with non-small cell lung cancer. Minerva Pneumologica. 2017;56:90-5.

14. Moshref Behzad N BN, Farzanegan B, Fathi M, Zareh Karizi S, Mohamadnia A. Expression of CK19-mRNA and CEA -mRNA biomarkers in pleural fluid of patients with non-small cell lung cancer. MINERVA PNEUMOLOGICA. 2017;56.

15. Li C, Xiong Y, Zhou Z, Peng Y, Huang H, Xu M, et al. Stereotactic body radiotherapy with concurrent chemotherapy extends survival of patients with limited stage small cell lung cancer: a single-center prospective phase II study. Medical Oncology. 2014;31(12):1-6.

16. González-Masiá JA, García-Olmo D, García-Olmo DC. Circulating nucleic acids in plasma and serum (CNAPS): applications in oncology. OncoTargets and therapy. 2013;6:819.

17. Quelle DE, Ashmun RA, Hannon GJ, Rehberger PA, Trono D, Richter KH, et al. Cloning and characterization of murine p16INK4a and p15INK4b genes. Oncogene. 
1995;11(4):635-45.

18. Romagosa C, Simonetti S, Lopez-Vicente L, Mazo A, Lleonart M, Castellvi J, et al. p16Ink4a overexpression in cancer: a tumor suppressor gene associated with senescence and high-grade tumors. Oncogene. 2011;30(18):2087-97.

19. Bitler BG, Goverdhan A, Schroeder JA. MUC1 regulates nuclear localization and function of the epidermal growth factor receptor. J Cell Sci. 2010;123(10):1716-23.

20. Brayman M, Thathiah A, Carson DD. MUC1: a multifunctional cell surface component of reproductive tissue epithelia. Reproductive Biology and Endocrinology. 2004;2(1):1.

21. Xu X, Bai L, Chen W, Padilla MT, Liu Y, Kim KC, et al. MUC1 contributes to BPDE-induced human bronchial epithelial cell transformation through facilitating EGFR activation. PloS one. 2012;7(3):e33846.

22. Kufe DW. Mucins in cancer: function, prognosis, and therapy. Nature Reviews Cancer. 2009;9(12):874-85.

23. TOUHIDI M, Keshmiri M, Ataran D, GHIASI MT, Azarian A. Tuberculus bronchostenosis presenting as anthracofibrosis. 2002.

24. Wynn GJ, Turkington PM, O'driscoll BR. Anthracofibrosis, bronchial stenosis with overlying anthracotic mucosa: possibly a new occupational lung disorder: a series of seven cases From one UK hospital. CHEST Journal. 2008;134(5):1069-73.

25. Pascolo L, Borelli V, Canzonieri V, Gianoncelli A, Birarda G, Bedolla DE, et al. Differential protein folding and chemical changes in lung tissues exposed to asbestos or particulates. Scientific reports. 2015;5:12129.

26. Wang H, Zheng J, Li Q, Zhou M, Ai D, Zhang H. Expression of the tumor suppressor gene p16, and lymph node metastasis in patients with ovarian cancer. Oncology Letters. 2017;14(4):4689-93.

27. CiesielskaU,Zatonski T, Nowinska K, Ratajczak-Wielgomas K, Grzegrzolka J, Piotrowska a, et al. Expression of Cell Cyclerelated Proteins p16, p27 and Ki-67 Proliferating Marker in Laryngeal Squamous Cell Carcinomas and in Laryngeal Papillomas. Anticancer Research. 2017;37(5):2407-15.

28. Kwon KY. Expression of MUC1 and MUC4 and its prognostic significance in non-small cell lung carcinoma. Korean Journal of Pathology. 2010;44:397-403.

This work is licensed under a Creative Commons AttributionNon Commercial 4.0 International License. 